\title{
Author Notes Provided by Corresponding Authors
}

\section{Xiao-hong Chen}

Xiao-hong Chen is a professor and doctoral supervisor at Central South University, and is at the same time working as the president of Hunan Institute of Commerce. Her research areas and interests include metal mineral resources utilization, and resource-conserving and environment-friendly engineering management, and decision theory.

This research was supported by the National Natural Science Foundation of China (71431006), and by the Ministry of Education Fund of Social Sciences (13JZD016).

Correspondence concerning this article should be addressed to Xiao-hong Chen, School of Business, Central South University, Changsha 410083, China. Email: cxh_csu@163.com

\section{Tao Li}

Tao Li is an associate professor, master supervisor at the School of Mechanical Engineering, Dalian University of Technology, Dalian, China. Her research interests include life cycle assessment of products, energy consumption of processes, sustainability of nanotechnology and product data management.

This research was supported by grants from the National Basic Research Program of China (Grant No. 2011CB013406).

Correspondence concerning this article should be addressed to Tao Li, School of Mechanical Engineering, Dalian University of Technology, Dalian 116023, China. E-mail: litao@dlut.edu.cn

\section{Wen-qiang Liu}

Wen-qiang Liu holds a Ph.D. in Management Science and Engineering from Institute of Nuclear Energy Technology of Tsinghua University. He used to engage in research of energy and resource in Tsinghua University. Then after, he used to work for State Economic and Trade Commission, National Development and Reform Commission, Ministry of Industry and Information Technology successively, and engaged in policy research and formulating and administration management. Currently, he is the vice-president of the Development Center of Information Industry of China (Institute of CCID, affiliated to the MIIT), and is mainly in charge of policy planning, research and consulting in manufacturing industry innovation, raw material industry, consumer goods industry, development of small and medium-sized enterprises, energy conservation and emission reduction, etc.

Correspondence concerning this article should be addressed to Wen-qiang Liu, the Development Center of Information Industry of China, Beijing 100048, China. Email: ccid@ccidgroup.com

\section{Xiao-qiu Shi}

Xiao-qiu Shi is a Ph.D. candidate at the School of Manufacturing Science and Engineering, Sichuan University. Shi's 
research areas and interests include intelligent planning and scheduling, and machine learning,

Correspondence concerning this article should be addressed to Xiao-qiu Shi, School of Manufacturing Science and Engineering, Sichuan University, Chengdu 610065, China. E-mail:315929207@qq.com

\section{Qiao Xiang}

Qiao Xiang, Master of Management and Doctor of Engineering, is a professor engineer in Chengdu Holy Industry \& Commerce Co. Ltd. (Group). Her research areas and interests include machinery and vehicle engineering management, aircraft engine maintenance technology and engineering management.

Correspondence concerning this article should be addressed to Qiao Xiang, Chengdu Holy Industry \& Commerce Co. Ltd. (Group), No.10, Gaopeng Avenue, Hi-tech Zone, Chengdu 610044, China. E-mail: xiangq@cae.cn; xiangqiao@vip.163.com

\section{Bin-shi Xu}

Bin-shi $\mathrm{Xu}$ is a professor at the National Priority Laboratory for Remanufacturing, Beijing. His research interests and areas include equipment maintenance engineering, surface engineering and remanufacturing engineering.

Correspondence concerning this article should be addressed to Bin-shi Xu, National Priority Laboratory for Remanufacturing, Beijing 100072, China. Email: xubinshi@vip.sina.com

\section{Hong-chao Zhang}

Hong-chao Zhang is the head of the working group on Life Cycle Assessment, Remanufacturing and Environmentally Benign Product Design and Manufacturing at the Institute of Sustainable Design and Manufacturing, Dalian University of Technology, and the interim chair and Derr Endowed Professor at the Department of Industrial Engineering, Texas Tech University, Lubbock, TX, USA. 